\title{
Long-Term Treatment with Telotristat Ethyl in Patients with Carcinoid Syndrome Symptoms: Results from the TELEPATH Study
}

\author{
Dieter Hörsch ${ }^{a}$ Lowell Anthony ${ }^{b}$ David J. Gross ${ }^{c}$ Juan W. Valle ${ }^{d}$ \\ Staffan Weline ${ }^{\text {e }}$ Marta Benavent ${ }^{f}$ Martyn Capling ${ }^{\text {Marianne Pavel }}{ }^{\text {h }}$ \\ Emily Bergsland ${ }^{i}$ Kjell Öberg ${ }^{e}$ Kenneth B. Kassler-Taub ${ }^{j}$ Polina Binder $^{j}$ \\ Phillip Banks ${ }^{j}$ Pablo Lapuertaj ${ }^{j}$ Matthew H. Kulkek
}

aDepartment of Gastroenterology/Endocrinology, Center for Neuroendocrine Tumors, Zentralklinik Bad Berka, Bad Berka, Germany; ${ }^{b}$ Markey Cancer Center, University of Kentucky, Lexington, KY, USA; 'Neuroendocrine Tumor Unit, Department of Medicine, Endocrine Service, European Neuroendocrine Tumor Society Center of Excellence, Hadassah-Hebrew University Medical Center, Jerusalem, Israel; dUniversity of Manchester/The Christie NHS

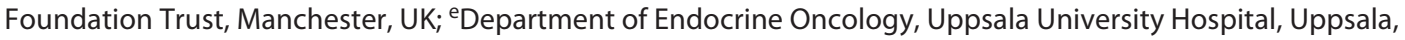
Sweden; ${ }^{f}$ Medical Oncology Department, Virgen del Rocío University Hospital/Instituto de Biomedicina de Sevilla, Seville, Spain; 9Department of Gastroenterology \& Hepatobiliary Medicine, Royal Free Hospital, Pond Street Centre for Gastroenterology, London, UK; 'hepartment of Gastroenterology and Hepatology, Charité-Universitätsmedizin, Berlin, Germany; 'Helen Diller Family Comprehensive Cancer Center, University of California at San Francisco, San

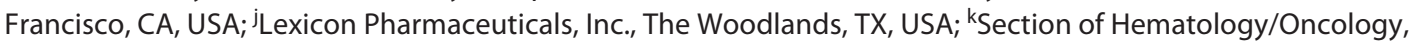
Boston University and Boston Medical Center, Boston, MA, USA

\section{Keywords}

Telotristat ethyl · Carcinoid syndrome · Diarrhea · Safety and tolerability · Quality of life

\begin{abstract}
Introduction: Telotristat ethyl is indicated for use in combination with somatostatin analogs (SSAs) to treat carcinoid syndrome (CS) diarrhea uncontrolled by SSAs alone in adults, but long-term safety and efficacy data beyond 48 weeks are needed. Objectives: The aims of the study were to evaluate the long-term safety and tolerability of telotristat ethyl and its effect on quality of life (QOL) in patients with CS. Methods: In this phase 3, nonrandomized, multicenter, open-label, long-term extension study (TELEPATH), patients who participated in phase 2 or 3 trials of telotristat ethyl contin-
\end{abstract}

karger@karger.com www.karger.com/nen

Karger $\stackrel{\text { ' }}{5}$

BOPEN ACCESS
(C) 2021 The Author(s)

Published by S. Karger AG, Basel

This is an Open Access article licensed under the Creative Commons Attribution-NonCommercial-4.0 International License (CC BY-NC) (http://www.karger.com/Services/OpenAccessLicense), applicable to the online version of the article only. Usage and distribution for commercial purposes requires written permission. ued treatment at their present dose level $(250$ or $500 \mathrm{mg}$ thrice daily) for 84 weeks. Safety and tolerability, the primary endpoint, were assessed by monitoring adverse events $(A E s)$, serious $A E s, A E s$ of special interest (AESIs; including liver-related $A E s$, depression, and gastrointestinal $A E s)$, and deaths. The secondary objective was to evaluate changes in patients' QOL using validated cancer questionnaires and a subjective global assessment of CS symptoms. Results: In 124 patients exposed to telotristat ethyl for a mean of 102.6 \pm 53.2 weeks, the type and frequency of AEs were consistent with those reported in previous trials. The occurrence of AESls was not related to dosage or duration of therapy. Most AEs were mild to moderate in severity, and no deaths were related to telotristat ethyl. QOL scores remained stable, and the majority of patients reported adequate symptom relief throughout the study. Conclusions: Safety results of TELE- 
PATH support the long-term use of telotristat ethyl in patients with CS diarrhea. Telotristat ethyl was well-tolerated and associated with sustained improvement in QOL scores (NCT02026063).

(c) 2021 The Author(s)

Published by S. Karger AG, Basel

\section{Introduction}

Up to one-fifth of patients with neuroendocrine tumors have carcinoid syndrome (CS), a condition characterized by flushing, diarrhea, and abdominal pain, among other symptoms [1]. Because neuroendocrine tumors are slow growing, patients may suffer from CS symptoms for years [2]. Diarrhea, which affects most patients, adversely affects activities of daily living, quality of life (QOL), and health outcomes [1-5]. Severe diarrhea may contribute to weight loss, electrolyte imbalance, and malnutrition, which in turn can contribute to increased morbidity and mortality as well as anxiety and social isolation $[6,7]$. In a Swedish survey, patients with CS reported higher levels of emotional distress, fatigue, and diarrhea, as well as worries about their prognosis, their families, and medical examinations than patients without CS [8]. Higher bowel movement frequency is strongly associated with more reports of fatigue, sleep disturbance, anxiety, depression, pain, and worsening social and physical function $[3,9]$. Interviews with patients participating in clinical trials of CS therapy also have documented negative effects of frequent bowel movements on emotional, social, physical, and occupational well-being $[10,11]$. Finally, CS not only places a heavy burden on patients but is also costly due to its comorbidities [12].

The overproduction of serotonin by neuroendocrine tumors contributes to the high incidence of diarrhea in CS [13]. Somatostatin analogs (SSAs), which inhibit serotonin secretion, are currently the standard of care for CS, but symptoms often reemerge over the course of this slowly progressing disease [13-17]. Telotristat ethyl is an orally administered tryptophan hydroxylase inhibitor indicated, at a dosage of $250 \mathrm{mg} 3$ times daily, for the treatment of CS diarrhea in combination with SSA therapy in adults whose symptoms are inadequately controlled by SSAs alone. The efficacy and safety of telotristat ethyl have been documented in a randomized, placebo-controlled, 4-week, ascending-multidose phase 2 study (NCT00853047); a 12-week, phase 2, dose-finding study (NCT01104415); and TELESTAR (NCT01677910) and TELECAST (NCT02063659), which were phase 3 trials that included a 12-week, double-blind, placebo-con- trolled period and an open-label extension period lasting an additional 36 weeks [18-21]. Evidence from these 4 studies supports not only reductions in CS symptoms but also modest improvements in patient-reported outcomes and weight $[10,11,22,23]$; however, long-term data are lacking. To fulfil this unmet need, a multicenter, longterm extension study (TELEPATH; NCT02026063) was conducted to evaluate the long-term safety and tolerability of telotristat ethyl as well as changes in QOL measures over 84 weeks.

\section{Materials and Methods}

This study was a nonrandomized, multicenter, open-label, long-term extension study to further evaluate the safety and tolerability of telotristat ethyl.

The study population included patients with CS who participated in a phase 2 or a phase 3 (TELESTAR or TELECAST) trial of telotristat ethyl, all of which have been previously described [1821 ]. For phase 2 study participants, institutional or local approval of the protocol was required prior to extension study entry (see suppl. appendix at www.karger.com/doi/10.1159/000516958). The parent trials and the extension study were conducted in accordance with Good Clinical Practice guidelines and the Declaration of Helsinki. Phase 3 study participants were invited to enter the extension study at the week 48 visit of the parent trial. All extension study participants provided written informed consent. Women of childbearing potential were required to use adequate contraception. Patients were excluded from extension study participation due to pregnancy, a major protocol violation during the parent trial, a medical condition that the investigator deemed a risk to patient safety, or institutionalization. Patients may also have been discontinued from the study if their CS worsened or another medical condition occurred whereupon the investigator decided that, in the interest of the patient, it was not medically acceptable to continue participation in the study.

Telotristat ethyl was given as 1 or 2250 -mg tablets ( 250 or 500 mg dose level, respectively) 3 times daily for $\geq 84$ weeks. In each country where the study was conducted, the study duration lasted until telotristat ethyl received regulatory approval to be marketed and available by prescription, or until June 30, 2018, whichever came first. Patients received the same dose level they received during the parent study, although if tolerability problems arose, the dose could be adjusted downward. Patients who experienced intolerability at the 250-mg dose level were discontinued from the study. Patients could continue taking background medications as long as dosages remained stable throughout the treatment period. Prescription or over-the-counter antidiarrheal medications, bile acid sequestrants, or pancreatic enzymes were allowed so long as these concomitant therapies were for a documented disease (e.g., fat malabsorption, bile acid malabsorption, or steatorrhea).

The primary objective of the study was to assess the long-term safety and tolerability of telotristat ethyl. Safety assessments included monitoring of adverse events (AEs), serious adverse events (SAEs), and deaths. In addition, clinical laboratory tests, vital sign measurements, physical examinations including weight measurements, and 12-lead electrocardiograms were conducted at each vis- 


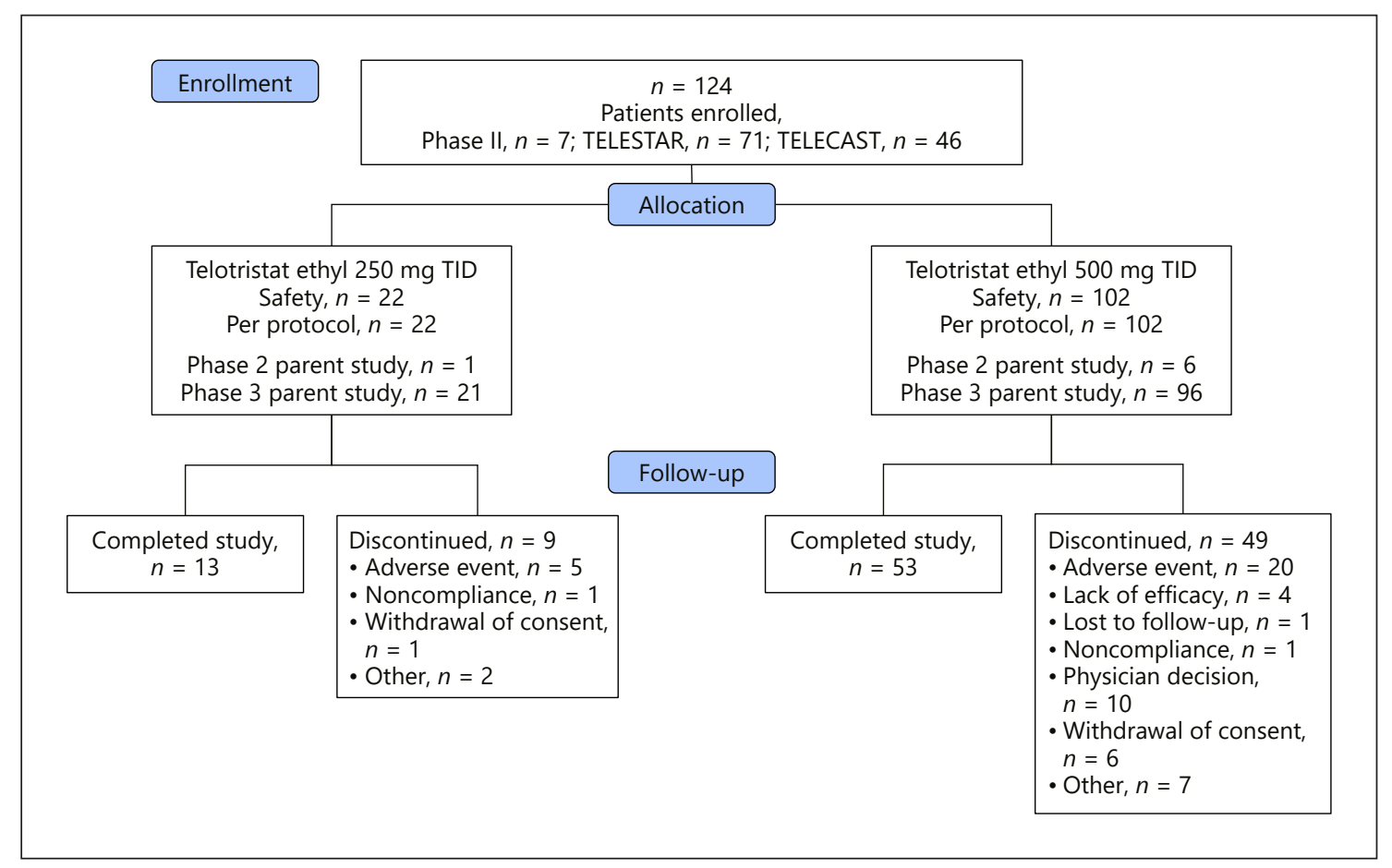

Fig. 1. Patient disposition. Patients entered the open-label extension on the same dose level of telotristat ethyl that they received during the parent trial. AE, adverse event; TID, 3 times daily.

it, and clinically significant changes from baseline in safety assessment variables were reported as AEs. A data safety monitoring board reviewed the safety data quarterly throughout the study and was responsible for monitoring AEs of special interest (AESIs), which included liver-related investigations, depression, and gastrointestinal (GI) symptoms. Liver enzyme elevations were evaluated according to Hy's law criteria [24]. Disease progression, which could be reported as an AE, was determined based on investigators' interpretation of clinical scans of the tumor (e.g., computed tomography, positron emission tomography, or magnetic resonance imaging) or the disease status assessed by the investigator.

The secondary objective of this study was to evaluate changes in patients' QOL through week 84 using the European Organisation for Research and Treatment of Cancer (EORTC) QLQ C30 and Gastrointestinal Symptoms of Carcinoid Neuroendocrine Tumors (GI.NET21) questionnaires and a subjective global assessment of CS symptoms $[25,26]$. The EORTC QLQ is designed to assess the health-related QOL of cancer patients participating in clinical trials. It includes 5 functional scales (physical functioning, role functioning, emotional functioning, cognitive functioning, and social functioning), 3 symptom scales (fatigue, nausea and vomiting, and pain), a global health status/QOL scale, and 6 single items (dyspnea, insomnia, appetite loss, constipation, diarrhea, and financial difficulties). Scores on each scale or item range from 0 to 100 , and higher scores represent a higher response level [25]. The GI.NET21 module includes 21 items comprising 5 scales and 4 single items and is also scored from 0 to 100 , with higher scores representing more severe symptoms [26]. In a subjective global assessment, at each visit, patients were asked whether they had experienced ade- quate relief of their CS bowel symptoms during the previous week and were also asked to rate the severity of CS symptoms on an 11 -point scale ranging from 0 to 10 , where 0 equaled "no symptoms" and 10 equaled the "worst symptoms ever experienced."

No formal sample size calculations were performed because the number of patients expected to participate in the study was estimated from the number of patients who participated in the parent trials. Analysis of safety data for the primary objective was based on the safety population, which included all patients who received any fraction of a dose of telotristat ethyl during the study. Statistical analysis of the safety data involved examination of the descriptive statistics and individual patient listings for any effects of study treatment on clinical tolerability and safety. Safety data were evaluated for concomitant medications, study drug exposure, treatment-emergent AEs, SAEs, deaths, physical examination findings, electrocardiogram results, clinical laboratory test results, and vital sign measurements.

The secondary objective was evaluated based on the per-protocol population, which consisted of patients who received telotristat ethyl and had no major protocol deviations that would interfere with the collection or interpretation of the QOL data. The analysis was based on observed data, and missing data were not imputed. The change from baseline in the overall and domain scores for the EORTC QLQ C30 and GI.NET21 questionnaires at each study visit was analyzed using a mixed model with repeated measures. The model was generalized to handle missing data and data specific to the measurement properties of the dependent variables. Nonparametric methods were used to supplement the tests and estimated from the mixed linear model. Analyses of QOL endpoints were 
Table 1. Baseline demographics and characteristics

\begin{tabular}{|c|c|c|c|}
\hline & $\begin{array}{l}\text { Telotristat } \\
\text { ethyl } 250 \mathrm{mg} \text { TID } \\
(n=22)\end{array}$ & $\begin{array}{l}\text { Telotristat } \\
\text { ethyl } 500 \mathrm{mg} \text { TID } \\
(n=102)\end{array}$ & $\begin{array}{l}\text { Total } \\
(N=124)\end{array}$ \\
\hline Mean age $\pm S D$, years & $64.5 \pm 7.6$ & $63.4 \pm 10.3$ & $63.6 \pm 9.9$ \\
\hline \multicolumn{4}{|l|}{ Sex, $n(\%)$} \\
\hline Male & $8(36.4)$ & $61(59.8)$ & $69(55.6)$ \\
\hline Female & $14(63.6)$ & $41(40.2)$ & $55(44.4)$ \\
\hline Child-bearing potential & $2(9.1)$ & $5(4.9)$ & $7(5.6)$ \\
\hline \multicolumn{4}{|l|}{ Race, $n(\%)$} \\
\hline White & $22(100)$ & $93(91.2)$ & $115(92.7)$ \\
\hline Black & 0 & $2(2.0)$ & $2(1.6)$ \\
\hline American Indian or Alaska Native & 0 & $1(1.0)$ & $1(0.8)$ \\
\hline Hispanic ethnicity, $n(\%)$ & 0 & $1(1.0)$ & $1(0.8)$ \\
\hline Mean weight $\pm \mathrm{SD}, \mathrm{kg}$ & $72.8 \pm 16.3$ & $77.1 \pm 16.7$ & $76.3 \pm 16.6$ \\
\hline \multicolumn{4}{|l|}{ Region of origin, $n(\%)$} \\
\hline North America & $4(18.1)$ & $34(33.3)$ & $38(30.6)$ \\
\hline Europe & $10(45.5)$ & $63(61.8)$ & $73(58.9)$ \\
\hline Rest of the world & $8(36.4)$ & $5(4.9)$ & $13(10.5)$ \\
\hline \multicolumn{4}{|l|}{ SSA use } \\
\hline Octreotide* & $9(40.9)$ & $71(68.6)$ & $80(63.7)$ \\
\hline Lanreotide & $9(40.9)$ & $25(24.5)$ & $34(27.4)$ \\
\hline \multicolumn{4}{|l|}{ Symptoms at entry } \\
\hline Diarrhea & $15(68.2)$ & $90(88.2)$ & $105(84.7)$ \\
\hline Flushing & $18(81.8)$ & $65(63.7)$ & $83(66.9)$ \\
\hline Abdominal pain & $8(36.4)$ & $37(36.3)$ & $45(36.3)$ \\
\hline Fatigue & $8(36.4)$ & $31(30.4)$ & $39(31.5)$ \\
\hline Insomnia & $3(13.6)$ & $26(25.5)$ & $29(23.4)$ \\
\hline Depression & $5(22.7)$ & $20(19.6)$ & $25(20.2)$ \\
\hline Carcinoid heart disease & $1(4.5)$ & $6(5.9)$ & $7(5.6)$ \\
\hline
\end{tabular}

SSA, somatostatin analog. ${ }^{*}$ One patient in the 500-mg dose level group used short-acting octreotide exclusively.

made using statistical tests, each with a 2 -sided $\alpha$ level $=0.05$. The least squares (LS) means, 2-sided 95\% confidence intervals (CIs), and the $p$ values of the LS means at each visit were reported to evaluate the within-patient treatment effects. The null hypothesis was that there was no treatment effect (no difference from baseline values) over time. Friedman's nonparametric method for testing treatment effect over time was used to supplement the mixed model with repeated measures. The null hypothesis of the test was that the change from baseline in a score was the same over time, and the alternate hypothesis was that the change from baseline in a score was different over time.

A generalized linear mixed model was used to evaluate the proportion of patients who reported adequate relief of CS symptoms at each study visit. The odds ratio versus baseline, its associated 2 -sided 95\% CIs, and $p$ value were reported for each post-baseline visit. The analyses of the change from baseline in subjective global assessment of CS symptoms on an 11-point scale were similar to that applied to the change from baseline in overall and domain scores of the EORTC QLQ C30 and GI.NET21 at each study visit. All statistical analyses were done using SAS (SAS Institute, Cary, NC, USA) statistical software version 9.3 or higher.

Long-Term Telotristat Ethyl Therapy in CS

\section{Results}

\section{Study Population}

The study was conducted at 45 sites in the United States, Canada, Europe, Israel, and Australia between January 14, 2014, and September 12, 2018 (online suppl. appendix). A total of 124 patients were enrolled in the study and included in the analysis (Fig. 1). At study entry, 102 patients were receiving telotristat ethyl 500 mg thrice daily, and 22 were receiving telotristat ethyl $250 \mathrm{mg}$ thrice daily. Seven patients entered the extension from a phase 2 parent study, and 117 entered from the 2 phase 3 parent trials. The majority of patients participating in the study were white and male with a mean age of 64 years (Table 1). Ages ranged from 36 to 89 years, with 3 patients $>80$ years of age at the time of consent. The mean weight at baseline was slightly higher in the 500-mg dose level group $(77.1 \pm 16.7$ vs. $72.8 \pm$ 
Table 2. Summary of treatment-emergent AEs

\begin{tabular}{lcr}
\hline & $\begin{array}{l}\text { Patients } \\
(N=124), \\
n(\%)\end{array}$ & \multicolumn{1}{l}{$\begin{array}{l}\text { Events, } \\
\end{array}$} \\
\hline Any AE & $122(98.4)$ & 1,173 \\
Treatment-related AE & $56(45.2)$ & 121 \\
Severe AE & $55(44.4)$ & 183 \\
SAE & $66(53.2)$ & 171 \\
Treatment-related serious AE & $3(2.4)$ & 3 \\
AE leading to study drug discontinuation & $22(17.7)$ & 36 \\
AE leading to study discontinuation & $25(20.2)$ & 32 \\
AE resulting in death & $20(16.1)$ & 20 \\
\hline
\end{tabular}

AE, adverse event; SAE, serious adverse event.

16.3), and more women than men received the $250-\mathrm{mg}$ dose (63 vs. $40 \%)$.

At the time of enrollment, patients had been diagnosed with metastatic neuroendocrine tumor for a median of 71.3 months. SSAs were used by the majority of patients at study entry (octreotide by 79 patients and lanreotide by 34 patients). Participating patients' symptoms at baseline were typical of CS. Diarrhea, flushing, abdominal pain, and fatigue were the most frequently reported baseline symptoms (Table 1). Diarrhea was more common at baseline in the $500-\mathrm{mg}$ than the 250 - mg group (88.2 vs. $68.2 \%$ ), whereas the 250 -mg group reported higher incidences of flushing (81.8 vs. 63.7\%) and constipation (22.7 vs. 5.9\%). Abdominal pain and fatigue were reported at similar frequencies across both dose level groups.

A total of 66 patients (53.2\%) completed the study and 58 patients $(46.8 \%)$ discontinued, of which 25 patients (20.2\%) did so due to an AE (Fig. 1), of which 3 patients had AEs that were considered related to telotristat ethyl (described below). Of the 33 patients who terminated the study early due to another reason (which included lack of efficacy, loss to follow-up, noncompliance, physician decision, and other reasons; see Fig. 1), 28 patients had at least 1 ongoing $\mathrm{AE}$ at the time of discontinuation. The majority of AEs were not related to study drug. A total of 9 events in 5 patients, all receiving the 500-mg dose level, that were ongoing at the time of study termination - but were not the reason for termination - were classified as possibly or probably related to study drug. Eructation, fatigue, hypertriglyceridemia, insomnia, and palpitations were all reported for 1 patient, and the following events occurred separately in 1 patient each: nausea, increased alanine aminotransferase (ALT), decreased appetite, and asthenia.
All patients entering the study had been exposed to telotristat ethyl for at least 9 months. Over the course of the extension study, the mean cumulative duration of treatment with any dose of telotristat ethyl was $102.6 \pm$ 53.2 weeks (median 106.2 weeks; range 1.4-234.0 weeks), amounting to 244.7 patient-years of exposure to telotristat ethyl. Seventeen patients (13.7\%) had $\geq 156$ weeks of exposure, and the longest duration of treatment was $\geq 228$ weeks in a single patient who was receiving the $250-\mathrm{mg}$ dose at study entry. The mean duration of treatment with the 250-mg dose level was $91.8 \pm 58.2$ weeks (median 104.0; range 1.7-234.0 weeks) and to $500 \mathrm{mg}$ was $105.0 \pm$ 52.1 weeks (median 112.7; range 1.4-202.3 weeks). The mean treatment compliance was $88.2 \%$.

\section{Primary Endpoint: Telotristat Ethyl Safety}

Adverse Events

Treatment-emergent AEs were reported in a majority of patients (98.4\%) (Table 2). Approximately one-fifth experienced an $\mathrm{AE}$ that led to telotristat ethyl discontinuation $(n=22[17.7 \%])$ or study discontinuation $(n=25$ [20.2\%]). Overall, 20 deaths (16.1\%) were reported, including 18 patients $(17.6 \%)$ who entered the study at the 500-mg dose level. Progressive disease and/or underlying malignancy was reported as the cause of death in 12 patients. Only 1 of the 20 deaths, a case of acute hepatic failure was considered by the investigator to be possibly related to study drug. However, the patient had tolerated telotristat ethyl $500 \mathrm{mg}$ for approximately 46 months before the onset of hepatic failure, which was assessed by the sponsor as unlikely to be related to study drug and attributable to the extensive involvement of the liver with carcinoid tumor ( $80 \%$ replacement) and the preexisting liver fibrosis (possibly due to alcohol). The other 7 deaths were deemed not related to study drug and included 3 deaths that were considered likely to be due to tumor progression, 1 each due to septic shock after a planned surgery, cardiopulmonary failure, intestinal perforation, and hemorrhagic stroke.

In general, the incidence of treatment-emergent AEs was similar between dose groups (Table 3). The most frequently reported AEs were GI disorders, including the preferred terms diarrhea, nausea, abdominal pain, constipation, and vomiting. Fatigue, pyrexia, disease progression, and peripheral edema were the most frequently reported general disorders and administration site conditions. The most frequently reported infections and infestations were nasopharyngitis and urinary tract infection. Decreases in appetite and weight were reported by 16 patients $(12.9 \%)$. 
Table 3. Incidence of treatment-emergent AEs experienced by $\geq 5 \%$ of study population by MedDRA system organ class and preferred term

\begin{tabular}{|c|c|c|}
\hline & $\begin{array}{l}\text { Patients } \\
(N=124), n(\%)\end{array}$ & $\begin{array}{l}\text { Events, } \\
n\end{array}$ \\
\hline Any AE & $122(98.4)$ & 1,733 \\
\hline Blood and lymphatic system disorders & $19(15.3)$ & 37 \\
\hline Anemia & $13(10.5)$ & 19 \\
\hline Cardiac disorders & $14(11.3)$ & 25 \\
\hline Endocrine disorders & $11(8.9)$ & 18 \\
\hline Eye disorders & $13(10.5)$ & 17 \\
\hline GI disorders & $96(77.4)$ & 448 \\
\hline Diarrhea & $44(35.5)$ & 89 \\
\hline Nausea & $41(33.1)$ & 68 \\
\hline Abdominal pain & $40(32.3)$ & 78 \\
\hline Constipation & $25(20.2)$ & 36 \\
\hline Vomiting & $25(20.2)$ & 38 \\
\hline Abdominal pain upper & $15(12.1)$ & 20 \\
\hline Flatulence & $8(6.5)$ & 8 \\
\hline General disorders and administration site conditions & $79(63.7)$ & 189 \\
\hline Fatigue & $32(25.8)$ & 41 \\
\hline Pyrexia & $21(16.9)$ & 26 \\
\hline Disease progression & $18(14.5)$ & 23 \\
\hline Edema peripheral & $18(14.5)$ & 20 \\
\hline Asthenia & $15(12.1)$ & 26 \\
\hline Hepatobiliary disorders & $20(16.1)$ & 31 \\
\hline Infections and infestations & $62(50.0)$ & 129 \\
\hline Nasopharyngitis & $11(8.9)$ & 18 \\
\hline Urinary tract infection & $11(8.9)$ & 12 \\
\hline Influenza & $10(8.1)$ & 10 \\
\hline Injury, poisoning, and procedural complications & $26(21.0)$ & 38 \\
\hline Investigations & $50(40.3)$ & 110 \\
\hline Weight decreased & $16(12.9)$ & 19 \\
\hline Metabolism and nutrition disorders & $44(35.5)$ & 90 \\
\hline Decreased appetite & $16(12.9)$ & 19 \\
\hline Dehydration & $7(5.6)$ & 7 \\
\hline Musculoskeletal and connective tissue disorders & $48(38.7)$ & 97 \\
\hline Back pain & $17(13.7)$ & 18 \\
\hline Arthralgia & $16(12.9)$ & 19 \\
\hline Musculoskeletal pain & $8(6.5)$ & 9 \\
\hline Flank pain & $7(5.6)$ & 8 \\
\hline Pain in extremity & $7(5.6)$ & 7 \\
\hline $\begin{array}{l}\text { Neoplasms benign, malignant, and unspecified (including cysts and } \\
\text { polyps) }\end{array}$ & $32(25.8)$ & 54 \\
\hline Neuroendocrine tumor & $7(5.6)$ & 14 \\
\hline Nervous system disorders & $54(43.5)$ & 96 \\
\hline Dizziness & $11(8.9)$ & 11 \\
\hline Headache & $11(8.9)$ & 14 \\
\hline Syncope & $11(8.9)$ & 14 \\
\hline Psychiatric disorders & $37(29.8)$ & 69 \\
\hline Depression & $13(10.5)$ & 18 \\
\hline Depressed mood & $9(7.3)$ & 15 \\
\hline Insomnia & $9(7.3)$ & 9 \\
\hline Renal and urinary disorders & $19(15.3)$ & 47 \\
\hline Reproductive system and breast disorders & $7(5.6)$ & 8 \\
\hline Respiratory, thoracic, and mediastinal disorders & $36(29.0)$ & 75 \\
\hline Dyspnea & $14(11.3)$ & 18 \\
\hline Cough & $12(9.7)$ & 12 \\
\hline Skin and subcutaneous tissue & $38(30.6)$ & 61 \\
\hline Pruritus & $8(6.5)$ & 8 \\
\hline Surgical and medical procedures & $7(5.6)$ & 22 \\
\hline Vascular disorders & $42(33.9)$ & 63 \\
\hline Flushing & $17(13.7)$ & 26 \\
\hline Hypertension & $14(11.3)$ & 17 \\
\hline
\end{tabular}

$\mathrm{AE}$, adverse event; GI, gastrointestinal. 
Table 4. Incidence of treatment-emergent AEs (by preferred term) considered related to telotristat ethyl and occurring in $\geq 2 \%$ of patients in the study population

\begin{tabular}{|c|c|c|}
\hline & $\begin{array}{l}\text { Patients } \\
(N=124), n(\%)\end{array}$ & $\begin{array}{l}\text { Events, } \\
n\end{array}$ \\
\hline Overall & $56(45.2)$ & 121 \\
\hline \multicolumn{3}{|l|}{ GI disorders } \\
\hline Diarrhea & $6(4.8)$ & 6 \\
\hline Nausea & $6(4.8)$ & 8 \\
\hline Abdominal pain & $3(2.4)$ & 3 \\
\hline Constipation & $7(5.6)$ & 11 \\
\hline \multicolumn{3}{|c|}{ General disorders and administration site conditions } \\
\hline Asthenia & $4(3.2)$ & 5 \\
\hline Fatigue & $8(6.5)$ & 8 \\
\hline Edema peripheral & $3(2.4)$ & 3 \\
\hline \multicolumn{3}{|l|}{ Investigations } \\
\hline $\begin{array}{l}\text { Gamma-glutamyltransferase } \\
\text { increased }\end{array}$ & $5(4.0)$ & 5 \\
\hline ALT increased & $3(2.4)$ & 3 \\
\hline Hepatic enzyme increased & $3(2.4)$ & 6 \\
\hline \multicolumn{3}{|c|}{ Metabolism and nutrition disorders } \\
\hline Decreased appetite & $5(4.0)$ & 6 \\
\hline \multicolumn{3}{|l|}{ Psychiatric disorders } \\
\hline Depression & $5(4.0)$ & 5 \\
\hline
\end{tabular}

$\mathrm{AE}$, adverse event; GI, gastrointestinal; ALT, alanine aminotransferase.

Table 4 lists AEs that were considered possibly or probably related to telotristat ethyl therapy. No such event occurred in more than 8 patients $(6.5 \%$ of the study population), and the most common related AEs were GI disorders (diarrhea, nausea, abdominal pain, and constipation). Other related AEs occurring in $>2$ patients included fatigue, decreased appetite, depression, asthenia, peripheral edema, and increases in gamma-glutamyltransferase, ALT, and unspecified hepatic enzyme. Diarrhea, nausea, and depression were reported with a similar incidence between the dose groups, but constipation, abdominal pain, and increases in gamma-glutamyltransferase and unspecified hepatic enzyme were reported more frequently by patients who entered the study at the 250 mg dose. Fatigue, decreased appetite, asthenia, peripheral edema, and increased ALT were reported more frequently in the 500-mg dose group. Hepatic enzyme elevations did not lead to significant clinical outcomes, and there were no cases meeting Hy's law criteria of hepatotoxicity.

Most AEs were mild to moderate in severity; only 5 possibly or probably related AEs (occurring in 5 patients) were considered severe: abdominal pain, prolonged co- agulation time, flank pain, hypertension, and acute hepatic failure. Patients who entered the study at the 500mg dose reported a higher incidence of severe AEs (48.0 vs. $27.3 \%)$ and AEs resulting in death (17.6 vs. 9.1\%). Patients recovered from all severe AEs except the case of acute hepatic failure, which was fatal (described above).

A total of 171 SAEs were reported by 66 patients (53.2\%), including 5 patients with 8 events whose preplanned hospitalizations were also reported as SAEs. The most frequently reported SAEs were disease progression (10.5\%), abdominal pain (4.8\%), and diarrhea (3.2\%). Patients who entered the study at the $250-\mathrm{mg}$ dose reported a higher incidence of treatment-related SAEs (9.1 vs. $1.0 \%)$. However, the incidence of SAEs (regardless of causal attribution to study drug) was similar between the 2 dose groups.

\section{Discontinuations}

AEs that led to telotristat ethyl discontinuation (but not study withdrawal) were reported by 22 patients $(17.7 \%)$ in similar proportions from the 2 dose groups; these included disease progression in 6 patients $(4.8 \%)$ and nausea, fatigue, and hepatic failure in 2 patients each (1.6\%). Both cases of hepatic failure had evidence of hepatic tumor progression. Of the 25 patients who withdrew from the study due to AEs, 3 patients reported 4 AEs that were considered possibly or definitely related to telotristat ethyl; 1 patient each reported fatal acute hepatic failure (described above), fatigue, and both toxic hepatitis and hepatic enzyme elevation. The other AEs leading to study discontinuation were considered unrelated to the study drug.

\section{AEs of Special Interest}

Liver-related AEs, depression, and GI AEs comprised the AESIs (Table 5). Liver-related AESIs were reported by 18 patients who experienced 28 events related to hepatic enzyme abnormalities; 16 of these patients had underlying metastatic neuroendocrine tumors in the liver and the other 2 had metastases in neighboring organs. The time to onset ranged from 1 to 793 days, and there was no apparent clustering. Three liver-related events were SAEs (hepatic enzyme elevation, abnormal liver function test, and hyperbilirubinemia). All events were mild or moderate in severity except 1 severe case of hyperbilirubinemia, which was not considered related to the study drug. Telotristat ethyl was discontinued for 2 patients after a liverrelated $\mathrm{AE}$ and temporarily stopped for another patient after a liver-related AE. Two patients had liver-related AEs that led to study withdrawal. 
Table 5. Adverse events of special interest

\begin{tabular}{|c|c|c|c|c|c|c|}
\hline & \multicolumn{2}{|c|}{$\begin{array}{l}\text { Telotristat ethyl } 250 \mathrm{mg} \\
\text { TID }(n=22)\end{array}$} & \multicolumn{2}{|c|}{$\begin{array}{l}\text { Telotristat ethyl } 500 \mathrm{mg} \\
\text { TID }(n=102)\end{array}$} & \multicolumn{2}{|c|}{ Total $(N=124)$} \\
\hline & $\begin{array}{l}\text { patients, } \\
n(\%)\end{array}$ & $\begin{array}{l}\text { events, } \\
n\end{array}$ & $\begin{array}{l}\text { patients, } \\
n(\%)\end{array}$ & events, $n$ & $\begin{array}{l}\text { patients, } \\
n(\%)\end{array}$ & events, $n$ \\
\hline Liver-related & $4(18.2)$ & 4 & $14(13.7)$ & 24 & $18(14.5)$ & 28 \\
\hline Depression-related & $2(9.1)$ & 5 & $24(23.5)$ & 37 & $26(21.0)$ & 42 \\
\hline Nausea & $5(22.7)$ & 19 & $36(35.3)$ & 49 & $41(33.1)$ & 68 \\
\hline Abdominal pain & $3(13.6)$ & 4 & $37(36.3)$ & 74 & $40(32.3)$ & 78 \\
\hline Constipation & $3(13.6)$ & 6 & $22(21.6)$ & 30 & $25(20.2)$ & 36 \\
\hline Vomiting & $1(4.5)$ & 6 & $24(23.5)$ & 32 & $25(20.2)$ & 38 \\
\hline Abdominal pain upper & $2(9.1)$ & 2 & $13(12.7)$ & 18 & $15(12.1)$ & 20 \\
\hline Flatulence & $1(4.5)$ & 1 & $7(6.9)$ & 7 & $8(6.5)$ & 8 \\
\hline GI pain & 0 & 0 & $2(2.0)$ & 2 & $2(1.6)$ & 2 \\
\hline GI sounds abnormal & 0 & 0 & $2(2.0)$ & 2 & $2(1.6)$ & 2 \\
\hline Abdominal discomfort & 0 & 0 & $1(1.0)$ & 1 & $1(0.8)$ & 1 \\
\hline Epigastric discomfort & 0 & 0 & $1(1.0)$ & 1 & $1(0.8)$ & 1 \\
\hline Noncardiac chest pain & 0 & 0 & $2(2.0)$ & 2 & $2(1.6)$ & 2 \\
\hline
\end{tabular}

A total of 42 depression-related AESIs were reported by 26 patients ( 11 males, 15 females; age $50-89$ years), 24 of whom were taking telotristat ethyl $500 \mathrm{mg}$ thrice daily at study entry. Fourteen of these patients entered the study with a history of depression, and approximately half were taking medication for the disease. Time to onset of depression events ranged from 2 to 953 days, and there was no apparent clustering around a given duration of treatment. All but 1 event were mild or moderate. Eleven of the 42 AESIs were reported as possibly related, and telotristat ethyl therapy was not altered in response to most events. In 1 case, the telotristat ethyl dose was reduced (which did not affect the patient's depression), and in another, telotristat ethyl therapy was interrupted (depression resolved). The duration of depression events ranged from 2 to 206 days; 13 events were ongoing at the end of the study.

A total of 369 GI-related AESIs were reported by 89 patients. Time to onset ranged from 1 day to approximately 45.5 months, with no apparent clustering around a specific time point. Most GI AEs lasted $\leq 2$ weeks, and the majority were mild to moderate in severity. Constipation was considered a particularly important AESI be- cause of the mechanism of telotristat ethyl. No fecaloma was reported. Of patients reporting constipation, 3 were taking the 250-mg dose and 22 the 500-mg dose when they entered the extension study. Most constipation events were nonserious of mild to moderate intensity. The single case reported as an SAE was assessed as not related to the study drug due to the patient's other medical conditions. It resolved after 2 days without a change in telotristat ethyl dosing. Two constipation events resulted in telotristat ethyl dose reductions.

\section{Secondary Endpoint: QOL Assessments}

The QLQ C30 and GI.NET21 remained stable through week 84 , with median changes from baseline through week 84 of 0 for all domains of the QLQ C30 and GI. NET21 scores. LS mean changes from baseline in both assessments were not statistically significant at any time point (Fig. 2).

Figure 3 shows the percentage of patients experiencing relief of GI-related CS symptoms over time. The odds ratio (OR) for patients reporting adequate relief appeared generally stable between week 12 (OR vs. baseline: 1.2 [95\% CI 0.6-2.3; $p=0.58$ ]; $n=106$ ) and week 84 (OR vs. 


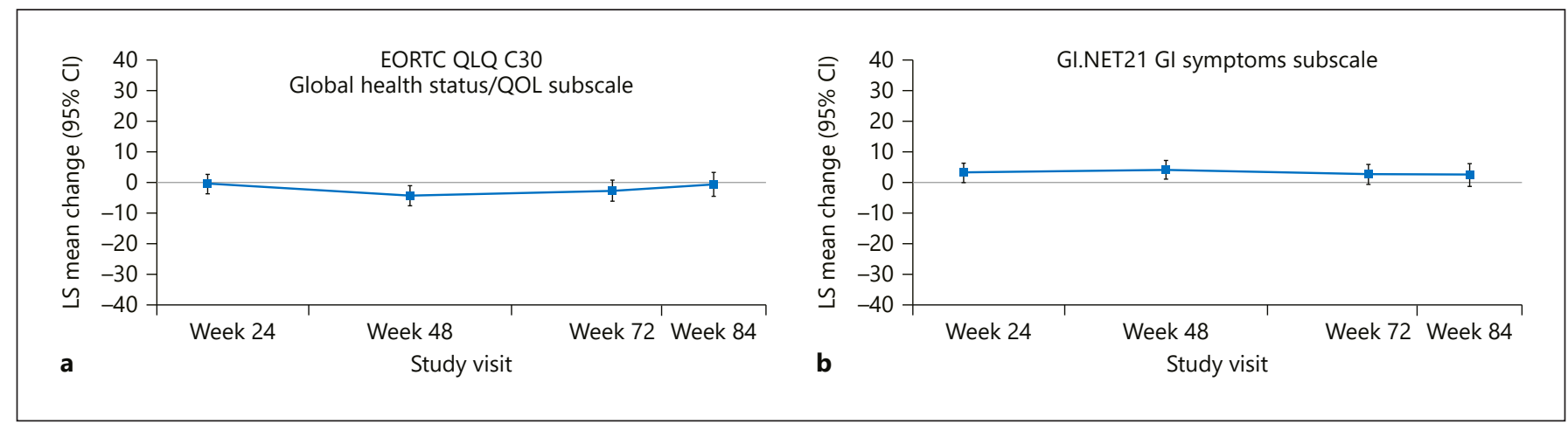

Fig. 2. LS mean change from baseline in global health status and QOL assessments of CS symptoms by study visit in the per-protocol population. a Global health status/QOL subscale of the EORTC QLQ C30. b GI symptoms subscale of the GI.NET21. Error bars represent 95\% CIs. EORTC, European Organisation for Research and Treatment of Cancer; GI.NET21, Gastrointestinal Symptoms of Carcinoid Neuroendocrine Tumors; LS, least square; QOL, quality of life; GI, gastrointestinal; CI, confidence interval; CS, carcinoid syndrome.
Fig. 3. Percentage of patients receiving telotristat ethyl who reported adequate relief of GI CS symptoms over the study period. GI, gastrointestinal; CS, carcinoid syndrome.

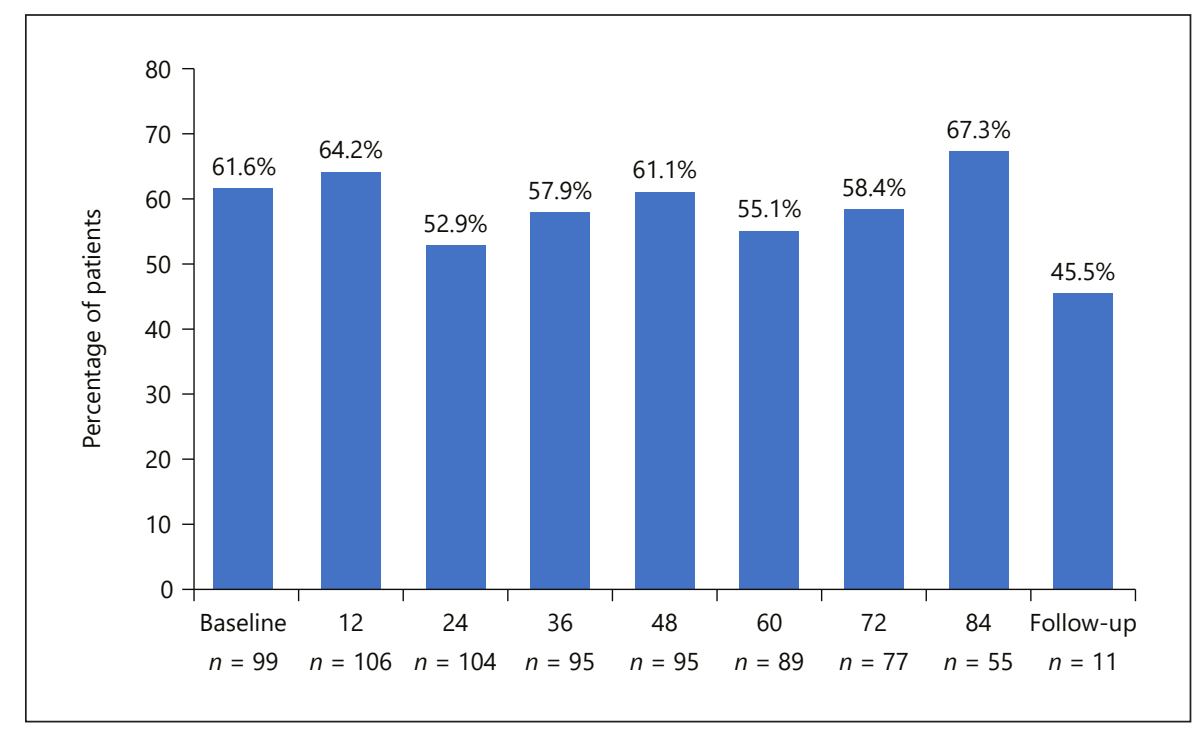

baseline: 1.5 [95\% CI: $0.7-3.4 ; p=0.33$ ]; $n=55$ ) relative to baseline, suggesting that the benefit obtained during the initial studies was maintained during TELEPATH. Concomitant therapy with antidiarrheal agents, intestinal anti-inflammatory agents, and intestinal anti-infective agents was reported by 25 patients overall (20.2\%), 23 of whom were receiving the $500-\mathrm{mg}$ dose of telotristat ethyl.

When asked about the severity of CS symptoms over the 7 days prior to each visit, patient responses remained generally stable over the entire study period. The mean severity scores ranged from $3.0 \pm 1.9$ at baseline to $3.4 \pm$ 2.3 at week 84 , with no significant changes over that period.

\section{Discussion/Conclusion}

In this long-term, open-label extension study, the safety of telotristat ethyl supported its use in patients with CS. Over $\geq 84$ weeks, telotristat ethyl maintained stable control of GI symptoms, with no deterioration in QOL scores or declines in the proportions of patients reporting adequate symptom relief throughout the extension study. The occurrence of AESIs, including liver-related AEs, depression, and GI symptoms, did not lead to changes in telotristat ethyl therapy in most patients. The incidence of constipation was low and was associated with few dosage reductions and no discontinuations of telotristat ethyl. 
Safety and tolerability was selected as the primary endpoint for this study to characterize the effects of longterm treatment with telotristat ethyl in patients with CS, a population that experiences a mean overall survival of 8-16 years [2]. The long-term exposure to CS-related symptoms weighs heavily on patients' overall QOL, and any new treatment used in this population must be not only safe and tolerable but must also sustain QOL. Our results suggest both criteria for long-term treatment were met. In this study, the cumulative exposure to telotristat ethyl averaged 2 years and was $>4$ years in some patients. In addition, modest improvements in QOL measures documented from the parent studies $[10,11$, 23] were maintained throughout the extension period. These results are supported by a recent pooled analysis of morbidity and mortality data from 239 participants in the phase 3 studies TELESTAR, TELECAST, and TELEPATH (the current phase 3 extension study reported here, which was ongoing at the time of data cut-off for the pooled analysis). Over a mean follow-up of 1.3 years and 309 total patient-years of exposure, no unexpected safety or tolerability concerns were observed, and no deaths were attributable to telotristat ethyl [27]. Nevertheless, patients should be monitored for safety and tolerability problems, particularly those taking concomitant medications associated with constipation (e.g., morphine).

Weight loss is frequently associated with uncontrolled CS and the severe diarrhea that often accompanies the disease, with increased morbidity and mortality [5-7]. Over the course of the 12-week TELESTAR trial, approximately one-third of patients receiving telotristat ethyl gained weight compared to only $5 \%$ of placebo-treated patients, and biochemical analyses suggested metabolic improvements in patients taking telotristat ethyl [22]. In the present extension study, weight loss was reported by $13 \%$ of study participants, suggesting that a large majority of patients may have retained improvements in nutritional status experienced during the parent trials.

The efficacy of telotristat ethyl grows over time, and by week 12 in TELESTAR, reductions in the frequency of bowel movements were greater with telotristat ethyl 500 $\mathrm{mg}$ than the 250-mg dose. The 500-mg dose also showed numerically greater effects on stool consistency, urgency, and use of short-acting octreotide rescue therapy. The proportion of patients achieving at least $3 \%$ weight gain during the double-blind treatment period was highest on $500 \mathrm{mg}(32.5 \%)$, followed by $250 \mathrm{mg}(17.1 \%)$, and placebo $(5.1 \%)[22]$.

Long-Term Telotristat Ethyl Therapy in CS
In the United States and Europe, telotristat ethyl is approved at a dose of $250 \mathrm{mg}$ thrice daily. Labeling decisions are focused on the primary endpoint of trials, rather than other measures such as those listed above. In TELESTAR, a third randomized treatment arm of telotristat ethyl 500 mg 3 times daily did not demonstrate additional treatment benefit on the primary endpoint and had a greater incidence of adverse reactions than the 250-mg 3 times daily dose. Therefore, regulatory authorities did not include telotristat ethyl $500 \mathrm{mg}$ as a recommended dose in the product label.

Approval of an orphan drug is typically granted with limited long-term safety data, which poses some uncertainty for potential safety issues. Here, the study results are generally reassuring. For example, in the TELESTAR study there was a short-term imbalance of depressionrelated $\mathrm{AEs}$, with more occurring in patients receiving the telotristat ethyl $500 \mathrm{mg}$ thrice daily than in those receiving placebo. The sample size was small, and the results were not repeated in another phase 3 study, TELECAST. The experience from the current study suggests that longer treatment is feasible without an increase in risk. This finding was in the context of regular monitoring, and regular follow-up of patients is needed to ensure that this clinical trial safety experience can be repeated in actual practice. From the efficacy perspective, a common question during the clinical development program was around the potential for tachyphylaxis. No such pattern was evident for telotristat ethyl, and long-term experience has continued in a meaningful number of patients with maintenance of symptom relief.

The study is limited by the lack of a control group and the study population, which likely represents patients who have tolerated telotristat ethyl and derived some benefit from their previous study participation. Nevertheless, assessment of additional long-term exposure to telotristat ethyl provided an opportunity to evaluate potential new significant risks in the setting of continued standard of care treatment. Another limitation is that only $53 \%$ of subjects completed the study. However, $<18 \%$ discontinued due to AEs.

In summary, long-term treatment with telotristat ethyl was generally well-tolerated and the clinical benefit appeared to be sustained. Safety findings were consistent with those of previous studies and raised no new concerns, thereby supporting the long-term safety of telotristat ethyl. The low incidence of weight loss and the durability of QOL in the patient population also support the long-term use of telotristat ethyl. 


\section{Acknowledgements}

We thank the patients, caregivers who participated in trials of telotristat ethyl, and all the study staff and personnel at study centers. Medical writing and editorial assistance was provided by Amanda Justice and Publication Practice Counsel, which was funded by Lexicon Pharmaceuticals, Inc.

\section{Statement of Ethics}

The parent trials and this extension study were conducted in accordance with Good Clinical Practice as required by the International Council for Harmonisation (ICH) guidelines and in accordance with country-specific laws and regulations governing clinical studies of investigational products and conformed with the ethical principles of the Declaration of Helsinki. The study protocol (LX1606.1 302 CS), all study protocol amendments, written study patient information, informed consent form, updates to these documents, and any other appropriate study-related information were reviewed and approved by the Institutional Review Board or Independent Ethics Committee /Ethics Review Committee at each study site (see online suppl. appendix) before the study began. All study participants provided written informed consent.

\section{Conflict of Interest Statement}

Dieter Hörsch has received personal fees and grants from Lexicon Pharmaceuticals, Inc.; Ipsen Pharmaceuticals, Inc.; Novartis Pharmaceuticals, Inc.; and Pfizer Pharmaceuticals, Inc., and advisory board honoraria from Advanz Pharma USA. Lowell Anthony has received honoraria for advisory boards from Lexicon, AbbVie, Curium, and Sun Pharma, and his institution has received grants from Lexicon and Entrinsic Health Solutions. David J. Gross has no financial interests to declare. Juan W. Valle has received personal fees from Agios, AstraZeneca, Debiopharm, Delcath Systems, Genoscience Pharma, Imaging Equipment Limited, Incyte, Ipsen, Keocyt, Merck, Mundipharma EDO, Novartis, PCI Biotech, Pieris Pharmaceuticals, QED, and Wren Laboratories, as well as grants, personal fees, and nonfinancial support from NuCana; personal fees and nonfinancial support from Pfizer; and grants and personal fees from Servier, all outside the submitted work. Staffan Welin has no financial interests to declare. Marta Benavent has received honoraria for advisory boards, meetings, and/or lectures from Novartis, Ipsen, Roche, and Pfizer. Martyn Caplin has received advisory board and speaker honoraria from AAA, Ipsen, Lexicon, Novartis, and Pfizer. Marianne Pavel has received honoraria for consultancy and presentations from Lexicon, Novartis, Ipsen, AAA, Boehringer Ingelheim, Pfizer, Keocyt, Riemser, and Prime Oncology and grants for research from Ipsen and Novartis. Emily Bergsland receives royalties from UpToDate. Kjell Öberg has no financial interests to disclose. Kenneth B. Kassler-Taub and Phillip Banks are employed by Lexicon Pharmaceuticals and hold stock in the company. Polina Binder and Pablo Lapuerta were employed by Lexicon Pharmaceuticals and held stock in the company at the time the research was conducted and the manuscript was written. Matthew H. Kulke has no financial interests to disclose.

\section{Funding Sources}

This study was supported and conducted by Lexicon Pharmaceuticals, Inc.

\section{Author Contributions}

D. Hörsch, L. Anthony, K. Oberg, M. Caplin, M. Pavel, P. Binder, P. Lapuerta., and M.H. Kulke participated in the design of the study and interpretation of the data. D. Hörsch, L. Anthony, D.J. Gross, J.W. Valle, M. Benavent, M. Caplin, E. Bergsland, S. Welin, M. Pavel, and M.H. Kulke participated in the conduct of the study and data collection and interpretation. P. Banks provided the statistical design of the trial; planned its analysis; and oversaw, reviewed, and approved the analysis results. P. Binder, P. Lapuerta, and K.B. Kassler-Taub oversaw the execution, data collection analysis, and reporting of the study. All authors made significant contributions in the development of the manuscript, including review, revision, and approval of the submitted manuscript.

\section{References}

1 Halperin DM, Shen C, Dasari A, Xu Y, Chu Y, Zhou $S$, et al. Frequency of carcinoid syndrome at neuroendocrine tumour diagnosis: a population-based study. Lancet Oncol. 2017;18(4):525-34.

2 Dasari A, Shen C, Halperin D, Zhao B, Zhou $\mathrm{S}, \mathrm{Xu} \mathrm{Y}$, et al. Trends in the incidence, prevalence, and survival outcomes in patients with neuroendocrine tumors in the United States. JAMA Oncol. 2017;3(10):1335-42.

3 Beaumont JL, Cella D, Phan AT, Choi S, Liu Z, Yao JC. Comparison of health-related quality of life in patients with neuroendocrine tumors with quality of life in the general US population. Pancreas. 2012;41(3):461-6.
4 Mocellin S, Nitti D. Gastrointestinal carcinoid: epidemiological and survival evidence from a large population-based study $(\mathrm{n}=25$ 531). Ann Oncol. 2013;24(12):3040-4.

5 Ter-Minassian M, Chan JA, Hooshmand SM, Brais LK, Daskalova A, Heafield R, et al. Clinical presentation, recurrence, and survival in patients with neuroendocrine tumors: results from a prospective institutional database. Endocr Relat Cancer. 2013;20(2):187-96.

6 Qureshi SA, Burch N, Druce M, Hattersley JG, Khan S, Gopalakrishnan K, et al. Screening for malnutrition in patients with gastroentero-pancreatic neuroendocrine tumours: a cross-sectional study. BMJ Open. 2016;6(5): e010765.
7 Maasberg S, Knappe-Drzikova B, Vonderbeck D, Jann H, Weylandt KH, Grieser C, et al. Malnutrition predicts clinical outcome in patients with neuroendocrine neoplasia. Neuroendocrinology. 2017;104(1):11-25.

8 Frojd C, Larsson G, Lampic C, von Essen L. Health related quality of life and psychosocial function among patients with carcinoid tumours. A longitudinal, prospective, and comparative study. Health Qual Life Outcomes. 2007;5:18.

9 Turaga KK, Kvols LK. Recent progress in the understanding, diagnosis, and treatment of gastroenteropancreatic neuroendocrine tumors. CA Cancer J Clin. 2011;61(2):113-32. 
10 Strosberg J, Joish VN, Giacalone S, Perez-Olle R, Fish-Steagall A, Kapoor K, et al. TELEPRO: patient-reported carcinoid syndrome symptom improvement following initiation of telotristat ethyl in the real world. Oncologist. 2019 Nov;24(11):1446-52.

11 Anthony L, Ervin C, Lapuerta P, Kulke MH, Kunz P, Bergsland E, et al. Understanding the patient experience with carcinoid syndrome: exit interviews from a randomized, placebocontrolled study of telotristat ethyl. Clin Ther. 2017;39(11):2158-68.

12 Broder MS, Chang E, Romanus D, Cherepanov D, Neary MP. Healthcare and economic impact of diarrhea in patients with carcinoid syndrome. World J Gastroenterol. 2016; 22(6):2118-25.

13 Strosberg JR, Halfdanarson TR, Bellizzi AM, Chan JA, Dillon JS, Heaney AP, et al. The North American Neuroendocrine Tumor Society consensus guidelines for surveillance and medical management of midgut neuroendocrine tumors. Pancreas. 2017;46(6):707-14

14 Oberg K, Lamberts SW. Somatostatin analogues in acromegaly and gastroenteropancreatic neuroendocrine tumours: past, present and future. Endocr Relat Cancer. 2016; 23(12):R551-r66.

15 Pavel M, Valle JW, Eriksson B, Rinke A, Caplin $\mathrm{M}$, Chen J, et al. ENETS consensus guidelines for the standards of care in neuroendocrine neoplasms: systemic therapy-biotherapy and novel targeted agents. Neuroendocrinology. 2017;105(3):266-80.
16 National Comprehensive Cancer Network. NCCN guidelines. neuroendocrine tumors of the GI, lung and thymus (Carcinoid Tumors); 2020. Available from: https://www.nccn.org/ professionals/physician_gls/default.aspx.

17 Pavel M, Öberg K, Falconi M, Krenning EP, Sundin A, Perren A, et al. Gastroenteropancreatic neuroendocrine neoplasms: ESMO clinical practice guidelines for diagnosis, treatment and follow-up. Ann Oncol. 2020; 31(7):844-60.

18 Pavel M, Hörsch D, Caplin M, Ramage J, Seufferlein T, Valle J, et al. Telotristat etiprate for carcinoid syndrome: a single-arm, multicenter trial. J Clin Endocrinol Metab. 2015; 100(4):1511-9.

19 Pavel M, Gross DJ, Benavent M, Perros P, Srirajaskanthan $\mathrm{R}$, Warner RRP, et al. Telotristat ethyl in carcinoid syndrome: safety and efficacy in the TELECAST phase 3 trial. Endocr Relat Cancer. 2018;25(3):309-22.

20 Kulke MH, O’Dorisio T, Phan A, Bergsland E, Law L, Banks P, et al. Telotristat etiprate, a novel serotonin synthesis inhibitor, in patients with carcinoid syndrome and diarrhea not adequately controlled by octreotide. Endocr Relat Cancer. 2014;21(5):705-14.

21 Kulke MH, Hörsch D, Caplin ME, Anthony LB, Bergsland E, Öberg K, et al. Telotristat ethyl, a tryptophan hydroxylase inhibitor for the treatment of carcinoid syndrome. J Clin Oncol. 2017;35(1):14-23.
22 Weickert MO, Kaltsas G, Hörsch D, Lapuerta $\mathrm{P}$, Pavel M, Valle JW, et al. Changes in weight associated with telotristat ethyl in the treatment of carcinoid syndrome. Clin Ther. 2018; 40(6):952-e2.

23 Gelhorn HL, Kulke MH, O’Dorisio T, Yang QM, Jackson J, Jackson S, et al. Patient-reported symptom experiences in patients with carcinoid syndrome after participation in a study of telotristat etiprate: a qualitative interview approach. Clin Ther. 2016;38(4):759-68.

24 Reuben A. Hy's law. Hepatology. 2004;39(2): 574-8.

25 Sprangers MA, Cull A, Bjordal K, Groenvold $\mathrm{M}$, Aaronson NK. The European Organization for research and treatment of cancer. Approach to quality of life assessment: guidelines for developing questionnaire modules. EORTC Study Group on Quality of Life. Qual Life Res. 1993;2(4):287-95.

26 Yadegarfar G, Friend L, Jones L, Plum LM, Ardill J, Taal B, et al. Validation of the EORTC QLQ-GINET21 questionnaire for assessing quality of life of patients with gastrointestinal neuroendocrine tumours. Br J Cancer. 2013; 108(2):301-10.

27 Anthony LB, Kulke MH, Caplin ME, Bergsland E, Öberg K, Pavel M, et al. Long-term safety experience with telotristat ethyl across five clinical studies in patients with carcinoid syndrome. Oncologist. 2019;24(8): e662-70. 